\title{
Addressing sight-distance-related safety effects of installing median barriers at horizontal curves of undivided highways under a 3D approach
}

\author{
César De Santos-Berbel ${ }^{1}$, Keila González-Gómez ${ }^{1}$, Maria Castro ${ }^{1}$ \& \\ Jose A. Anta ${ }^{2}$ \\ ${ }^{1}$ Department of Civil Engineering-Transport and Territory, Technical University of \\ Madrid, Spain. ${ }^{2}$ Environmental Systems Research Institute (ESRI), Spain
}

\begin{abstract}
Concrete barriers are often installed to separate opposing directions of traffic at hazardous horizontal curves on undivided highways in order to avoid frequent types of crashes such as head-on collisions. Whereas this treatment is effective for preventing head-on crashes, they reduce the available sight distance (ASD). The estimation of sight distance is usually performed under a two-dimensional (2D) approach, despite the three-dimensional (3D) nature of facilities. Radius curve, clearance and trajectory location on cross section are the only variables considered under the planar approach, ignoring the spatial component. To overcome this limitation, this study applies a 3D sight distance estimation method, where the sight distance reduction caused by installing barriers is evaluated, contemplating their shape and position in a detailed fully $3 D$ model. The procedure facilitates incorporating mass standardized constructive elements into the highway model. This method consists of a geoprocessing model on a geographic information system (GIS), where the impact of the terrain, the roadway itself, and the road furniture on sight distance is broached. The results evidenced that the ASD outcomes were different under either approach. In addition, the ASD fell below the required stopping sight distance (SSD) of the standards under either approach. A potential application of this method is the analysis of the overall safety effects and the subsequent evaluation of crash modification factors associated to this safety measure.
\end{abstract}

Keywords: traffic safety, sight distance, median barriers, 3D modelling, geographic information system

\section{Introduction}

Sight distance is a fundamental factor to account for in highway design and operation. During highway operation safety issues may arise, requiring countermeasures that reduce the frequency or severity of collisions. The use of concrete barriers to separate opposing directions of traffic is usually motivated by 
a high risk of head-on collisions and off-road excursions. However, the possible adverse effects of the introduction of this safety measure, in particular on the sight distance, should be taken into account. No relevant additional hazard should be created after the implementation of countermeasures.

To achieve an accurate estimation of sight distance on in-service facilities is difficult if an appropriate method is lacking. The most common difficulties involve the use of two-dimensional (2D) models instead of three-dimensional (3D) models, inaccurate 3D highway recreation, and the overlooking of structures and roadside elements.

The main objective of this study is to apply a 3D method to evaluate sight distance where barriers are present and assess their potential effects on safety. In addition, the sight distance results of the 3D method proposed are compared with the sight distance results obtained under a 2D approach.

\section{Background}

The available sight distance (ASD) is defined as the distance, measured along the vehicle path, between the driver's position and the farthest target visible on such a path without sight obstruction. The geometric design of the highway must enable sufficient sight distance along the entire route. Most highway standards and guides propose 2D models to estimate the ASD. On the horizontal projection, sight distance is typically restricted by obstructions by the inner roadside. International standards and guides provide models to ensure sufficient sight distance on curves as a function of the available clearance between the driver's trajectory and the sight obstruction [1,2]. However, this approach ignores the 3D nature of highways.

The estimation of sight distance must contemplate alignment combinations on both projections and 3D features. Indeed, significant differences arise between 2D and 3D sight distance results on different 3D alignment combinations [3]. Several 3D methods are available to estimate sight distance where the highway and its roadsides were modelled by means of finite elements [3-5]. GIS-based applications can likewise be used to compute 3D sight distance on highways [6]. Sightlines are evaluated on an elevation model, typically derived from a light detection and ranging (LiDAR) survey, where the sight obstructions are identified at ease [7]. However, the use of elevation models from different sources for highway sight distance studies may also lead to differences in the sight distance outcome [8].

Barriers can be relevant sight restrictions located outside the travelled way where they separate opposing directions of traffic, particularly on left curves overlapped with a crest. In some cases, the theoretical stopping sight distance (SSD) can only be attained if the target height rises [9]. However, the decision of safety improvements must not depend on rather discretionary design assumptions. The criteria for the provision of sight distance around barriers and structures, and the effects of the design output on construction cost, speed and safety must be carefully considered [10].

The effect of roadside sight restrictions on safety issues has been addressed by several studies. The probability of collision caused by median barriers limiting sight 
distance was modelled using reliability theory [11]. In addition, the probability of collision due to limited sight distance on curves was found to be a significant variable in safety performance functions [12]. Moreover, higher accident rates were reported on left curves of motorways due to sight restrictions on medians such as barriers or pillars [13].

\section{Methods and materials}

The effect of placing concrete barriers on the ASD has been studied through two procedures: a 3D method and a 2D method based on standardized assumptions.

First, a geoprocessing model on GIS was used to compute sight distance in 3D [14]. The geoprocessing model launches sightlines repeatedly from each driver's location, namely stations, on the vehicle path and forward. The algorithm checks whether the sightlines are obstructed or not, determining the sightline output and the ASD from each station on the path, which were spaced $5 \mathrm{~m}$ apart.

In addition to the driver's path, the geoprocessing model requires an elevation model to enable the evaluation of sightlines. Both digital terrain models (DTM) and digital surface models (DSM) are supported. DTMs are elevation models that represent the topography of the bare ground surface, whereas DSMs comprise relevant landscape elements above the terrain in addition to the bare ground. The due formats of the elevation models can be either a raster file or a triangulated irregular network (TIN) dataset. The elevation model used in this study consisted of a surface built up from a point cloud arranged in a 1-m grid derived from an airborne LiDAR survey.

The procedure facilitates incorporating mass standardized constructive elements into the highway model as additional sight obstructions. These additional modelling inputs must be included as a multipatch feature class (or shapefile) in the GIS. A multipatch feature is a GIS object that stores a collection of patches to represent the boundary of a $3 \mathrm{D}$ object [15]. The due interchange file format to insert these features in GIS is Collaborative Design Activity (CollaDA), which can be created and handled by many 3D modelling applications once known their dimensions. The main advantage of this modelling method is that it allows the insertion of elements that are not necessarily found in reality or that are not properly recreated by the elevation model into the virtual 3D model, thus making it possible to evaluate the effect of including new road equipment or additional structures on sight distance.

Second, a 2D method for the estimation of the ASD was employed. On the circular arc of a curve, and assuming that the observer and the target that define the sightline are located on the same position of their respective cross sections, the ASD can be estimated according to the Spanish standard [1] as per Eq. 1:

$$
A S D=2 \cdot R \cdot \operatorname{acos}\left(\frac{F+R}{R+b}\right)
$$

The variables in Eq. (1) are shown in Figure 1a. $F$ is the clearance of the sight obstruction by the roadside, $R$ is the curvature radius of the inner lane edge, and $b$ is the offset between the inner lane edge and the driver's position on the lane (trajectory offset), which is assumed to match the target point offset. 
According to the Spanish standard [1], the SSD must be checked on every lane of the cross section. Therefore the sight distance estimation was launched three times under both the 2D and the 3D assumption. On the vertical projection, it also determines that the height of the observer's point above the pavement surface is $1.1 \mathrm{~m}$ and that of the target is $0.5 \mathrm{~m}$. These values, along with the barrier height, result in the layout shown in Figure $1 \mathrm{~b}$, where the middle point of the sightline has the same height than the barrier. Thus, slight variations in the geometric features may produce important differences in the sight distance outcome.
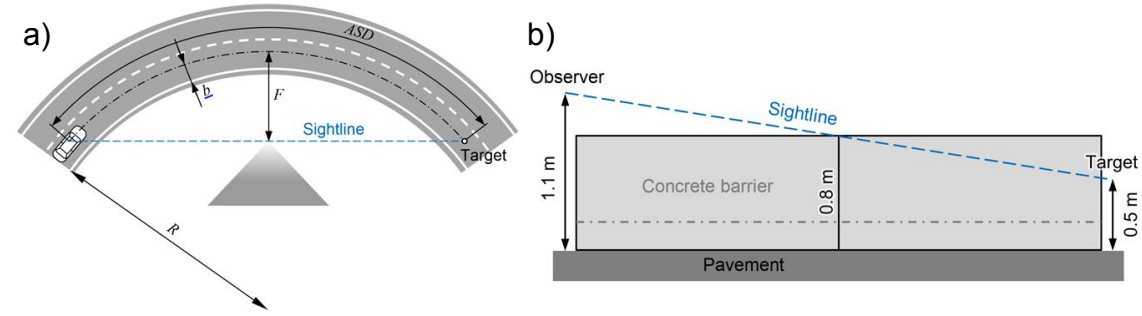

Figure 1. a) 2D Sight distance estimation on horizontal projection according to Spanish standard [1], b) Sketch of sightline profile and concrete barrier.

In order to evaluate the potential safety effects of insufficient sight distance, the ASD and the required SSD must be compared. The latter is defined as the distance needed by a driver forced to stop a vehicle facing an unexpected obstacle on its path. The SSD can be determined according to the expression provided by the Spanish standard [1], which reads:

$$
S S D=\frac{V \cdot t}{3.6}+\frac{V^{2}}{254 \cdot(f+g)}
$$

where $V$ is the speed, $t$ is the perception and reaction time (2 seconds), $f$ is the friction factor and $g$ is the grade.

A highway section located near Guadalajara (Spain) was selected for the assessment, its design speed being $100 \mathrm{~km} / \mathrm{h}$. The horizontal alignment in this section is composed of a sequence of reverse curves which runs through a composite profile on a hilly area, where no modification of the highway geometry could be proposed at a reasonable cost. On the vertical alignment, the long and steep grade $(5.8 \%)$ that features the section can increase considerably the operating speed of vehicles driving downhill. This creates potential safety issues on right turns, as a lane departure could become a head-on collision on an undivided section. For this reason, concrete barriers were installed on the highway centreline at a right turn in the downhill direction. In addition, concrete barriers were also installed on the inner shoulder of the same curve, next to a near-vertical cutside slope, to prevent landslide chunks from invading the roadway. The radius of such a curve is $650 \mathrm{~m}$, which is preceded by a curve of radius $450 \mathrm{~m}$. The highway cross section comprises one lane in the outward direction (downhill) and two lanes in the return direction (uphill). The lane width is $3.5 \mathrm{~m}$, and the median width is 1.5 $\mathrm{m}$. The 3D virtual model was built up according to these data. Figure $2 \mathrm{a}$ illustrates the actual view of the sub section where the concrete barriers were installed, and Figure 2b illustrates the 3D virtual recreation of the highway in ArcScene. 


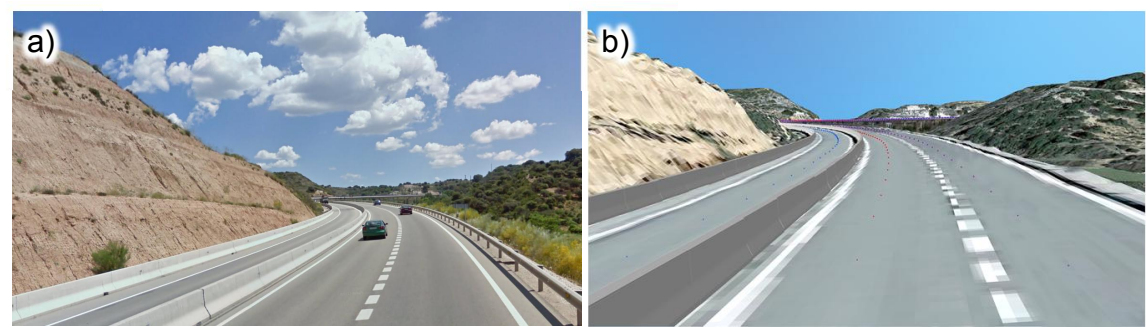

Figure 2. a) View of the section with concrete barriers (source: Google Street View), b) 3D Scene generated in ArcScene.

\section{Results and discussion}

In this section, the sight distance outcome of the 2D and the 3D methods are presented and compared. Given the geometric parameters above described and the layout of the barriers, the ASD can be estimated in 2D. The position of the nearvertical face of the barrier is considered for the value of the clearance in $2 \mathrm{D}$. The results of this estimation are provided in Table 1.

Table 1. 2D sight distance estimation.

\begin{tabular}{|c|c|c|c|c|}
\hline Lane & $\mathrm{R}[\mathrm{m}]$ & $\mathrm{F}[\mathrm{m}]$ & $\mathrm{b}[\mathrm{m}]$ & $\mathrm{ASD}[\mathrm{m}]$ \\
\hline Outward direction & 645.75 & 1.2 & 2 & 128.4 \\
\hline Inner, return direction & 650.75 & 0.65 & 1.5 & 105.7 \\
\hline Outer, return direction & 654.25 & 4.15 & 1.5 & 171.9 \\
\hline
\end{tabular}

A more detailed analysis and a comparison of results in shown in the following subsections. It must be noted that each direction has independent stationing.

\subsection{Outward direction}

In this direction, the concrete barrier by the inner roadside spans from station $5+505$ to $5+985$, while the median barrier was installed between stations $5+665$ and $6+215$. Figure 3 displays the sight distance results in the outward direction, where the dashed blue line represents the ASD under a 3D approach in the scenario without barriers, and the solid blue line displays the ASD outcome after the installation of concrete barriers. The results of the 3D estimation showed two areas where a reduction of the ASD is produced owing to the presence of the concrete barriers. The concrete median barrier reduced the ASD from beginning to station $5+490$. This could hardly be predicted under a 2D approach because the sight obstruction is produced on the transition curves that lay in the middle of the reverse curve. From station 5+500 to 5+830, the concrete barrier by the roadside was the obstruction that reduced the ASD. According to Table 1, the ASD on the circular arc of the curve, considering the barrier by the inner roadside under a 2D approach, was $128.4 \mathrm{~m}$, and ranges from station $5+620$ to $5+885$. This zone is displayed by the dotted red line. Significant differences arose between the outputs of the 
estimated 2D ASD and the estimated 3D ASD considering the barrier. First, the locations where minimum sight distance was predicted by the $2 \mathrm{D}$ approach and where they were actually produced did not match. Second, the 2D estimation method underestimated the minimum ASD. These differences were both due to the positive effect of superelevation and the precision achieved with the 3D modelling. Since the observer and target locations have a more favourable elevation in comparison to the concrete barrier, more sightlines run above the concrete barrier without being cut off. Also, both the elevation model and the precise barrier modelling enabled more reliable results.

The ASD sets were compared with the required SSD. Given the geometric features of the highway section, the required SSD was $205.8 \mathrm{~m}$ as per Eq. (2). Each of the three set falls below this value at some point, even in the case of 3D ASD without barriers. Under the 3D approach with barriers, the minimum ASD was $150 \mathrm{~m}$, which remains significantly below the required SSD.

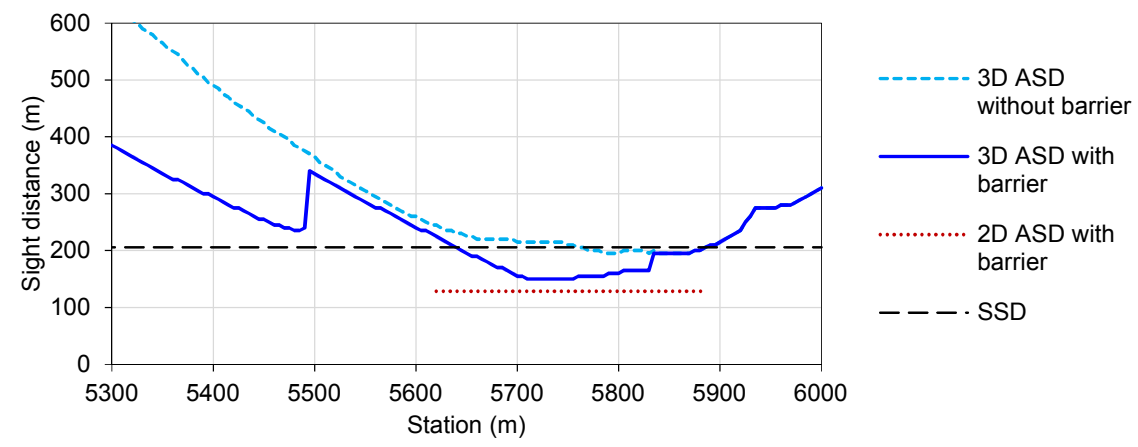

Figure 3. Sight distance comparison in the outward direction.

\subsection{Return direction}

In the return direction, the cross section comprises two lanes, and the sight distance was estimated on both lanes under the 2D and the 3D approaches. The median barrier spans from station $1+325$ to $1+925$. The concrete barrier installed by the inner roadside was found not to affect the ASD.

As shown in Figure 4, the 3D ASD resulted greater than the 2D ASD on both lanes. When considering the median barrier, the results on the inner lane presented a less significant difference than those of the outer lane. Moreover, on the outer lane, the predicted location of the reduced ASD caused by the barrier under the 2D approach (dotter yellow line) was far from matching the results of the 3D method (solid green line). Conversely, the zones with minimum ASD under either approach almost coincided on the inner lane, whose trajectory is closer to the barrier. Therefore, the results under the 2D assumption provided less accuracy as the clearance increases owing to the positive effect of the superelevation. Unlike in the outward direction, the 3D ASD complied with the standard before installing the barrier. After it was installed, potential sight-distance-related issues are more likely to arise on the inner lane, whereas the ASD on the outer lane meets by far the SSD. 


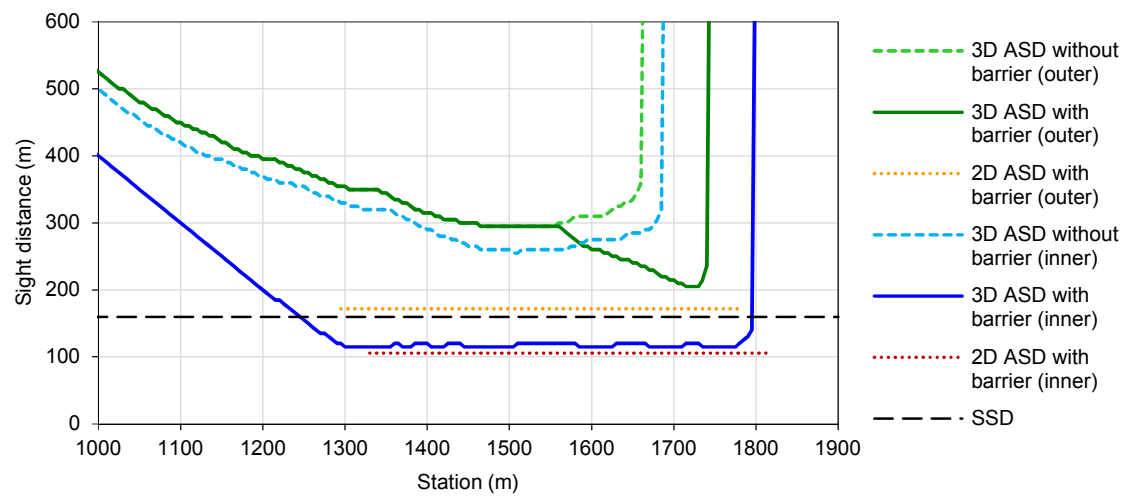

Figure 4. Sight distance comparison in the return direction.

\section{Conclusions}

This paper presents a 3D method to address sight-distance-related safety effects of installing median barriers at horizontal curves of undivided highways. The 3D approach permitted to consider the 3D nature of the highway and the features outside the travelled way, it delivering a more reliable outcome. In addition, the capability of the modelling method to include fully-3D elements provides high accuracy and a reliable risk assessment tool.

The reduction of the ASD owing to the concrete barriers according to the $3 D$ estimation method was less pronounced than that predicted under the $2 \mathrm{D}$ approach. On a horizontal curve, the 2D method underestimated the ASD because of the favourable effect of superelevation. In addition, the stretch affected by the ASD reduction was shorter than that predicted under the $2 \mathrm{D}$ approach. The favourable effect of superelevation increases as the lateral clearance and the superelevation rate increase. Although barriers reduce the ASD, they provide positive visual guidance on the highway alignment. Therefore, the potential negative effect on safety of installing concrete barriers created by the reduction of the ASD is compensated to a certain extent. In addition, it should be borne in mind that other operational effects may arise on single lanes enclosed between two continuous barrier strips.

Future lines of research contemplate the study of operating speeds in the section and the consideration of different vehicle types in the assessment. In addition, the development of statistical studies, calibration of collision modification factors, and the analysis of the overall safety effects of this safety measure will be pursued.

\section{Acknowledgements}

The authors gratefully acknowledge the financial support of the Spanish Ministerio de Economía y Competitividad and European Regional Development Fund (FEDER). Research Project TRA2015-63579-R (MINECO/FEDER). 


\section{References}

[1] Ministerio de Fomento. Norma 3.1-IC: Trazado, Ministerio de Fomento, 2016.

[2] American Association of State Highway and Transportation Officials (AASHTO). A Policy on Geometric Design of Highways and Streets, sixth edition, AASHTO, 2011.

[3] Ismail, K., Sayed, T.: New Algorithm for Calculating 3D Available Sight Distance. Journal of Transportation Engineering, 133, pp. 572-581, 2007.

[4] Hassan, Y., Easa, S.M., Abd El Halim, A.O.: Analytical Model for Sight Distance Analysis on Three-Dimensional Highway Alignments. Transportation Research Record, 1523, pp. 1-10, 1996.

[5] Hassan Y., Easa S., Abd El Halim A.O.: Design Considerations for Combined Highway Alignments. Journal of Transportaion Engineering, pp. 60-68, 1997.

[6] Castro M., Anta J.A., Iglesias L., Sánchez J.A.: GIS-Based System for Sight Distance Analysis of Highways. Journal of Computing in Civil Engineering, 28, 04014005, 2014.

[7] Khattak, A.J., Shamayleh, H.: Highway Safety Assessment through Geographic Information System-Based Data Visualization. Journal of Computing in Civil Engineering, 19, pp. 407-411, 2005.

[8] Castro, M., Lopez-Cuervo, S., Paréns-González, M., De Santos-Berbel, C.: LIDAR-Based Roadway and Roadside Modelling for Sight Distance Studies. Survey Review, 48, pp. 309-315, 2016.

[9] Mavromatis, S., Mertzanis, F., Kleioutis, G., Psarianos, B.: Three-Dimensional Stopping Sight Distance Control on Passing Lanes of Divided Highways. European Transport Research Review, 8, pp. 1-10, 2016.

[10]Arndt, O.K., Cox, R., Lennie, S.C., Whitehead, M.T.: Provision of Sight Distance around Concrete Barriers and Structures on Freeways and Interchanges. Transportation Research Record, 2262, pp. 22-30, 2011.

[11]Hussein, M., Sayed, T., Ismail K., Van Espen, A.: Calibrating Road Design Guides Using Risk-Based Reliability Analysis. Journal of Transportation Engineering, 140, 04014041, 2014.

[12] Ibrahim, S.E., Sayed, T.: Developing Safety Performance Functions Incorporating Reliability-Based Risk Measures. Accident Analysis \& Prevention, 43, pp. 2153-2159, 2011.

[13]Krüger, D., Weinert, R., Weiser, F.: Sicherheits wirkung geringer Sichtweiten in Linkskurven auf Autobahnen. Forschung Straßenbau und Straßenverkehrstechnik, 886, 2004.

[14]Iglesias, L., Castro, M., Pascual, V., De Santos-Berbel, C.: Estimation of sight distance on highways with overhanging elements. International Conference on Traffic and Transportation Engineering, pp. 75-82, Belgrade, Serbia, November 24-25, 2016.

[15]Environmental Systems Research Institute (ESRI). Multipatches - Help | ArcGIS for desktop, http://desktop.arcgis.com/en/arcmap/latest/extensions/3d-analyst/multipatches. $\mathrm{htm}$, 06.03.2018. 